\title{
Die Musikpflege am Münchener kurfürstlichen Hof im Spiegel der historischen Musikalienbestände der
} Bayerischen Staatsbibliothek.

\section{Ein Beitrag zur Sammlungs- und Überlieferungsgeschichte}

Die große Bedeutung der Überlieferung der höfischen Musik des 18. Jahrhunderts in der Sächsischen Landesbibliothek - Staats- und Universitätsbibliothek Dresden wird durch den Vergleich mit erhaltenen Musikaliensammlungen ebenbürtiger Fürstenhäuser besonders deutlich. Aufschlussreich ist ein Blick auf die Bestände der Bayerischen Staatsbibliothek in München, die das musikalische Erbe der Wittelsbacher Kurfürstenfamilie verwahrt. Seit der Doppelhochzeit des sächsischen Kurprinzen Friedrich Christian mit Prinzessin Maria Antonia Walpurgis und seiner Schwester Maria Anna (1728-1797) mit dem bayerischen Kurfürsten Maximilian III. Joseph (1727-1777) im Jahr 1747 bestanden enge familiäre Bindungen zwischen den beiden Höfen, die nicht ohne Auswirkungen auf den Musikalienaustausch blieben. ${ }^{1}$

Da der ältere Teil der in Dresden erhaltenen privaten Musikaliensammlung der Kurfürstin Maria Antonia noch Quellen aus ihrer Münchener Zeit umfasst, lassen sich dabei auch interessante inhaltliche Überschneidungen zwischen den beiden Sammlungen beobachten. Zudem brach der enge Kontakt der Kurfürstin zum Hof ihres Bruders in späteren Jahren nicht ab, wie wir durch das Zeugnis von Charles Burney erfahren, der die musikliebende Fürstin auf ihrer Rückreise aus Italien 1772 in München traf. So erlebte er sie als Interpretin eigener Kompositionen in einem Kammerkonzert in Nymphenburg an der Seite ihres Gambe spielenden Bruders. ${ }^{2}$

Im folgenden Überblick soll zunächst die im 19. Jahrhundert vollzogene Transformation der Musikaliensammlung der Königlichen Hofbibliothek in die Musikabteilung der Bayerischen Staatsbibliothek beschrieben werden, bevor einige Besonderheiten der Münchener Überlieferung des höfischen Musikrepertoires des 17. und 18. Jahrhunderts diskutiert werden. Dabei wer-

1 Verwandtschaftliche Verhältnisse bestanden schon früher, da die Gattinnen der Kurfürsten Karl Albrecht und Friedrich August II. beide Töchter Kaiser Josephs I. waren. Dies führte jedoch noch nicht zu einem wesentlichen kulturellen Austausch zwischen den beiden Höfen.

2 Charles Burney, The Present State Of Music In Germany, The Netherlands, And United Provinces. Or The Journal of a Tour Through Those Countries, Undertaken to Collect Materials for A General History Of Music, Vol. 1, London ${ }^{2}$ 1775, S. 140. Zu diesem Zeitpunkt hielt sich auch Johann Gottlieb Naumann in München auf. 
den zunächst die beiden wichtigsten Teilbereiche der Kirchenmusik und des Opernrepertoires betrachtet, worauf auch die erhaltenen Reste von Privatmusikalien aus dem Besitz von Mitgliedern der kurfürstlichen Familie vorgestellt werden. Die in Dresden durch die Schrank-II-Sammlung so prominent repräsentierte Instrumentalmusik des 18. Jahrhunderts ist in München nur sehr lückenhaft überliefert, aus der ersten Hälfte des Jahrhunderts sind sogar überhaupt keine nennenswerten handschriftlichen Quellen erhalten, so dass dieser Aspekt nicht näher behandelt werden muss.

Die Bayerische Staatsbibliothek - vormals Münchener Königliche Hofbibliothek - beheimatet eine der ältesten eigenständigen Musiksammlungen in Deutschland. Ein erstes Verzeichnis der Musikalien wurde bereits 1802 von dem Bibliothekssekretär Johann Baptist Bernhart erstellt (D-Mbs: Cod Bav.Cat. 220 b). Damals waren ältere Bestände der Hofkapelle, die nicht mehr benötigt wurden, an die Hofbibliothek abgegeben worden, was zu ihrer Inventarisierung führte. ${ }^{3}$ Das noch heute gebräuchliche akzessorische Signatursystem wurde 1814 für die Drucke (Signaturgruppe Mus.pr.) und 1828/29 für die Handschriften (Mus.ms.) eingeführt, als der Hofprediger und Musikaliensammler Johann Michael Hauber (1778-1843) einen detaillierten sechzehnbändigen thematischen Katalog anlegte (D-Mbs: Cbm Cat 270 / 270a). Zwischen 1802 und 1828 muss es demnach zu zahlreichen weiteren Abgaben von Hofkapellnoten an die Königliche Bibliothek gekommen sein. Einen weiteren Zuwachs erfuhr die Sammlung durch die Übernahme von Noten aus dem Besitz Haubers, der Eigentümer einer umfangreichen Musikaliensammlung war, darunter auch Partituren, die sich ursprünglich im Besitz der Kurfürstin Maria Anna befunden hatten, etwa Josef Mysliwiceks Oratorium Isacco figura del rendentore (Signatur Mus.ms. 246). ${ }^{4} 1857$ erhielt die Bibliothek eine eigene Musikabteilung. In diesem Rahmen wurde das Repertorium von dem ersten Kustos dieser Abteilung Julius Joseph Maier (1821-1889) systematisch ausgebaut und fortgeführt. Maier schaffte als Vereinfachung die von Hauber vorgesehene Unterteilung in Formatgruppen ab, so dass er bei einigen älteren Handschriften im Oktavformat Umsignierungen vornehmen musste. ${ }^{5} \mathrm{Im}$ Jahr der Gründung der Musikabteilung gelangten auch zahlreiche ältere Musikalien aus dem Besitz des Hofkapellmeisters Karl Aiblinger (1779-1867) in den Besitz der Bibliothek, darunter weitere Handschriften aus der ehemaligen Sammlung der Kurfürstin Maria Anna. ${ }^{6}$

Im Jahr 1860 wurde noch einmal ein großer Bestand an Musikalien, die nicht mehr für das aktuelle Musizieren benötigt wurden, von der Königlichen Hofmusikintendanz an die Musikabteilung abgegeben, darunter auch noch zwölf Partituren von italienischen Opern des späten

3 Helmut Hell, Vorwort zu: Bayerische Staatsbibliothek, Katalog der Musikhandschriften 1: Chorbücher und Handschriften in chorbuchartiger Notation (Kataloge Bayerischer Musiksammlungen. 5/1), München 1989, S. 21*-24*.

4 Dies betraf nur einzelne Stücke der Hauber-Sammlung, deren größter Teil in das Archiv der Theatiner-Hofkirche St. Kajetan gelangte. Dieser Bestand wird heute ebenfalls in der Musikabteilung der Bayerischen Staatsbibliothek aufbewahrt.

5 Siehe hierzu Hartmut Schäfer, „Bestandsgruppen und Formalkataloge in der Musikabteilung der Bayerischen Staatsbibliothek“, in: Bibliotheksforum Bayern, 20,2 (1992), S. 137-153.

6 Auch im Besitz Haubers befanden sich einige Musikalien aus dem Nachlass Maria Annas, darunter die Partitur des Oratoriums Isacco figura del redentore von Josef Mysliveček (Mus.ms. 246). Zu dieser Sammlung vgl. Gertraut Haberkamp und Robert Münster, Die ehemaligen Musikhandschriftensammlungen der Königlichen Hofkapelle und der Kurfürstin Maria Anna in München. Thematischer Katalog (Kataloge Bayerischer Musiksammlungen. 9). 
18. Jahrhunderts, die als Karnevalsopern für das Hoftheater unter Kurfürst Karl Theodor entstanden waren, sowie zahlreiche Kirchenkompositionen des 18. Jahrhunderts.

Eine besonders fortschrittliche musikbibliothekarische Leistung stellt Maiers gedruckter Katalog der älteren Musikhandschriften dar, in dem die Handschriften des 16. und 17. Jahrhunderts erfasst sind, darunter neben den Chorbüchern auch Tabulaturen, Stimmbücher, sowie Sammelhandschriften mit Claviermusik und vokaler Kammermusik. ${ }^{7}$

\section{Höfische Kirchenmusik}

Herzstück des in der Bayerischen Staatsbibliothek erhaltenen musikalischen Erbes der Wittelsbacher bilden weniger die Quellen des 18. Jahrhunderts - die ja den besonderen Wert der Dresdner Sammlung ausmachen -, sondern vor allem die in Chorbüchern notierten Werke des 16. Jahrhunderts aus der für die Münchener Musikgeschichte so glorreichen Ära der Kapellmeister Ludwig Senfl und Orlando di Lasso. Die wertvollsten dieser Quellen wurden über zwei Jahrhunderte in der fürstlichen Privatbibliothek aufbewahrt, da sie persönliches Eigentum der Wittelsbacher waren. Charles Burney konnte die alten Handschriften in der Residenz einsehen, ihr außergewöhnlicher Wert fiel dem musikhistorisch interessierten Reisenden sofort auf: „The library of the Elector is more rich in old musical authors, and in old compositions, than any one that I have yet seen in Europe“ ${ }^{8}$ Es handelt sich bei diesen Chorbüchern um teilweise aufwändig und kunstvoll illuminierte Prachthandschriften, die als Geschenke oder Auftragsarbeiten für Angehörige der herzoglichen Familie angefertigt wurden. Die beiden bekanntesten Beispiele sind die Codices mit Motetten von Cipriano de Rore (Mus.ms. B) und mit den Bußpsalmen von Orlando di Lasso (Mus.ms. A), beide verziert mit den berühmten Illuminierungen von Hans Mielich.

Die für den alltäglichen Gebrauch angefertigten Chorbücher befanden sich dagegen im Bestand der Hofkapelle. Sie wurden in der oben beschriebenen Weise zwischen 1795 und 1830 an die Bibliothek abgegeben. Die ältesten Exemplare stammen aus der Zeit Ludwig Senfls, darunter die Proprienvertonungen von Heinrich Isaac und Senfl von 1531 (Mus.ms. 35-38) und die Fortsetzung der Vertonung des Commune sanctorum, an der Senfl bis zu seinem Tod 1541 arbeitete (Mus.ms. 30). Senfl hatte sich zum Ziel gesetzt, für München ein Repertoire aufzubauen, das sich an dem Vorbild der Wiener Hofkapelle Maximilians I. auf Grundlage der Werke seines Lehrers Heinrich Isaac orientierte.

1550 erfolgte der Regierungsantritt Herzog Albrechts V., dem Gründer der Bibliothek. Unter ihm wurde Orlando di Lasso 1557 als Tenor der Hofkapelle angestellt, von 1564 bis zu seinem Tod 1594 bekleidete er als Nachfolger Ludwig Dasers das Amt des Kapellmeisters. Er trat zunächst vor allem mit weltlicher Musik hervor, ab ca. 1562 beherrschte er dann auch das liturgische Repertoire. $\mathrm{Ab} 1580$ bedeutete die Einführung des römischen nachtridentinischen Ritus am Münchener Hof

7 Julius Josef Maier, Die Musikalischen Handschriften der K. Hof- und Staatsbibliothek in München, Teil 1: Die Handschriften bis zum Ende des 17. Jahrhunderts (Catalogus codicum manuscriptorum bibliothecae regiae monacenis, tom. 8, 1), München 1879.

8 Burney, The Present State Of Music (wie Anm. 1), S. 130. 
eine stärker an römischen Vorbildern geprägte Neuausrichtung des Repertoires, was wiederum zu zahlreichen in Chorbüchern überlieferten Neukompositionen Lassos führte. ${ }^{9}$

Der bereits 1603 verstorbene Nachfolger Lassos, Johannes a Fossa, ist der letzte ältere Komponist, der in der Münchener Chorbuchsammlung vertreten ist. In den folgenden Jahrzehnten wurde das aus der Ära Lassos überkommene Repertoire weiter gepflegt, so dass keine Neukompositionen benötigt wurden. Erst für 1669 ist mit Johann Caspar Kerlls fünfstimmigem Requiem (Mus. ms. 67) wieder ein Werk eines Münchener Hofkapellmeisters in Chorbuchnotation nachweisbar. Unter seinen Nachfolgern, dem aus Rom stammenden Ercole Bernabei (1622-1687) und seinem Sohn Giuseppe Antonio (1649-1732), die für fast siebzig Jahre die Kirchenmusik am Münchener Hof prägten sollten, wurden wieder regelmäßig neue Werke im stile antico römischer Prägung komponiert. Noch 1715 wurden sechs vierstimmige a-capella-Messen Giuseppe Antonio Bernabeis in Chorbuchnotation aufgeschrieben. ${ }^{10}$

Während dieses traditionelle liturgische Repertoire relativ gut überliefert ist, sind von der konzertierenden und instrumental begleiteten Kirchenmusik des 17. Jahrhunderts, die in Stimmen notiert wurde, keine Quellen aus dem Hofkapellbestand erhalten. Heute ist von diesem älteren Repertoire im ,stile moderno fast nur noch Musik bekannt, die in zeitgenössischen Drucken veröffentlicht wurde, etwa die 1621 unter dem Titel Musicali melodie erschienenen Motetten und Canzonen von Giovanni Martino Cesare, die Cantiones sacrae von Johann Caspar Kerll von 1669 oder Ercole Bernabeis Sacrae modulationes op. II, die 1691 posthum erschienen. Die Werke Giuseppe Antonio Bernabeis im Stil der klassischen Vokalpolyphonie, vom Ende des 17. Jahrhunderts bis in das erste Viertel des 18. Jahrhunderts entstanden, sind dagegen in großer Zahl im ehemaligen Bestand der Hofkapelle überliefert, der in der 1837 von Ludwig I. erbauten Allerheiligen-Hofkirche aufbewahrt wurde. Bernabei galt als bedeutender Vertreter der prima pratica und hielt sich deshalb so bemerkenswert kontinuierlich im Repertoire der Hofkapelle. Zahlreiche der erhaltenen Stimmen sind originales Material, das unter der Leitung des Komponisten von dem Hofnotisten Gottlieb Sebastian Reischberger angefertigt wurde. Auch einige Autographe Bernabeis finden sich noch in diesem Bestand, darunter der vollständige Stimmensatz seines Stabat mater in g-moll von 1715 (Mus.ms. Mk 290), eines der wenigen erhaltenen Werke Bernabeis mit obligater Instrumentalbegleitung. ${ }^{11}$ Nach der kriegsbedingten Auslagerung in die Theatinerkirche St. Kajetan - gerade noch rechtzeitig vor der Zerstörung der Allerheiligen-Hofkirche am 18. März 1943 - werden diese Bestände heute zusammen mit den übrigen Musikalien der Theatinerkirche, darunter die umfangreiche Sammlung Johann Michael Haubers, in der Musikabteilung der Bayerischen Staatsbibliothek verwahrt.

Das älteste erhaltene Inventar der Kirchenmusikalien der kurfürstlichen Hofkapelle, der Catalogus rerum ad musicam spectantium, et quae pro usu in Capella Electorali servatur, wurde 1753, nach dem Regierungsantritt Kurfürst Max III. Josephs, auf Anordnung des neuernannten Inten-

9 Helmut Hell, Vorwort zu: Bayerische Staatsbibliothek, Katalog der Musikhandschriften 1 (wie Anm. 2), S. $13^{\star}-20^{*}$.

10 Mus.ms. 2754 „6 Missae alla Capell[a]“.

11 Geschichte und Umfang des Bestandes sind beschrieben im Vorwort zu Gmeinwieser, Die Musikhandschriften in der Theatinerkirche St. Kajetan in München. Thematischer Katalog (Kataloge Bayerischer Musiksammlungen. 4), München 1979. 
danten der Hofmusik Joseph Anton Graf von Seeau angelegt. ${ }^{12}$ In diesem Katalog sind auch die noch immer in Gebrauch befindlichen Chorbücher eingetragen, sie sind dort unter der Überschrift „Coral e Contrapunct libri“"verzeichnet. Konzertierende Figuralmusik des frühen und mittleren 17. Jahrhunderts wird hingegen nicht mehr aufgeführt, ältester namentlich benannter Komponist ist Giuseppe Antonio Bernabei. Vermutlich wurde das ältere Repertoire schon früher ausgesondert, da es nicht mehr als zeitgemäß empfunden wurde, womit die oben beschriebenen Repertoirelücken zu erklären wären. Das Inventar enthält ausschließlich die Musik Münchener Komponisten, neben den Werken der Kapellmeister Giuseppe Antonio Bernabei, Pietro Torri und Giovanni Porta auch Kompositionen von anderen Mitgliedern der Hofkapelle wie Bernardo Aliprandi, Antonio Casati, Johann Anton Berberich (Perprich) und Giacomo Bertolotti. Der Import von Werken auswärtiger Komponisten, etwa im Vergleich zu den zahlreichen im Bestand der Katholischen Hofkirche in Dresden nachweisbaren Werken Antonio Caldaras, aber auch von verschiedenen neapolitanischen und venezianischen Meistern, kann in München nicht beobachtet werden, hier verließ man sich ganz auf die Produktionen einheimischer Komponisten. Ähnlich wie in Dresden gehörten auch in München Instrumentalwerke zum kirchenmusikalischen Repertoire. Kurze Instrumentalkompositionen in orchestraler Besetzung wurden als Gradualmusiken verwendet, Pastoralsinfonien kamen während der Weihnachtsgottesdienste zur Aufführung. Unter den Komponisten dieser Kirchensinfonien finden wir die Konzertmeister Melchior d'Ardespin und Wenzel Wodiczka, aber auch die Kapellmeister Bernabei und Torri sowie den Kurfürsten Max III. Joseph persönlich. Letzterer betätigte sich ähnlich wie seine Dresdner Schwester Maria Antonia als Komponist, wobei er im Gegensatz zu dieser vor allem Kirchen- und Instrumentalmusik schrieb. Einige seiner Triosonaten und Sinfonien sind auch in der Dresdner Schrank-II-Sammlung in Abschriften Johann Georg Pisendels überliefert, ${ }^{13}$ aus der Sammlung Maria Antonias sind zudem zwölf Sinfonien in Abschriften eines Münchener Hofnotisten erhalten. ${ }^{14}$ In München ist dagegen nur noch eine einzige Sinfonie des Kurfürsten vorhanden. ${ }^{15}$ Interessanterweise fehlt in dem Verzeichnis ein Hinweis auf Kompositionen des bis 1755 in München tätigen Kammerkomponisten Giovanni Ferrandini, des Lehrers von Maria Antonia und Max Joseph. Seine Instrumentalwerke und seine italienischen Passionskantaten scheinen demnach nicht zum offiziellen kirchenmusikalischen Repertoire gehört zu haben. Vermutlich wurden sie ausschließlich zu privaten Anlässen, etwa bei Kammerkonzerten und in Privatandachten, aufgeführt.

Ab 1806, nach der Erhebung Bayerns zum Königreich, begann man mit einem neuen, detaillierteren Verzeichnis der Hofkapellbestände, das bis weit ins 19. Jahrhundert fortgeführt wurde. Der 442 Blätter umfassende Catalog über sämtliche Kirchenmusik, welche sich im Magazin der königl. Hofkapelle befindet, ${ }^{16}$ verzeichnet die Werkeinträge nun auch mit Incipits. Hauptschreiber ist der seit 1804 als Hoftheaterkopist angestellte Joseph Anton Schweigenberger. Der für die Erforschung des Repertoires der Hofkapelle so außergewöhnlich wertvolle Katalog gelangte erst

Vollständig wiedergegeben bei Haberkamp und Münster, Die ehemaligen Musikhandschriftensammlungen (wie Anm. 6), S. X-XVI.

13 Vergleiche unter anderem das Konvolut mit 12 Sinfonien, D-Dl: Mus.3261-N-13.

14 D-Dl: Mus.2-N-9,1-12 (Partitur und Stimmen eines Münchener Hofnotisten) / Mus.2-N-9,1a.

15 D-Mbs: Mus.ms. 512.

16 D-Mbs: Mus.ms. 10193. Vgl. Haberkamp und Münster, Die ehemaligen Musikhandschriftensammlungen (wie Anm. 6), S. XV-XVIII. Der Katalog wurde in diesem Band ediert. 
1971 in den Besitz der Bayerischen Staatsbibliothek und war der Forschung zuvor völlig unbekannt. Interessanterweise entfielen hier die Einträge zahlreicher Werke, die in dem Katalog von 1753 noch nachgewiesen waren, darunter sämtliche Kompositionen Pietro Torris (er war im älteren Katalog noch mit 70 Kompositionen vertreten) sowie die Werke der ,kleineren' Münchener Komponisten Aliprandi, Cassati, Berberich und Portolotti. Auch die oben genannten Kirchensinfonien fehlen in dem neueren Verzeichnis, an ihre Stelle tritt aber eine erhaltene Sammlung mit neueren Sinfonien Wodickas. Von Bernabei wurden fast nur Werke in reiner Vokalbesetzung mit Basso continuo aufbewahrt, während etwa seine konzertierenden Messen (der Katalog von 1753 nennt allein 14 Messvertonungen „con tubis“, also mit festlicher Trompetenbegleitung) fehlen; im Katalog von 1806 sind nur noch acht Messen im a-capella-Stil aufgeführt. Genannt werden in dem Katalog auch Partituren und Stimmen zu Opern und Oratorien, darunter nicht nur die in München aufgeführten Werke, sondern auch für auswärtige Theater und Höfe entstandene Kompositionen, etwa Johann Christian Bachs Londoner Serenata Endimione sowie zahlreiche Hasse-Opern. Vermutlich wurden aus diesen Materialen auch einzelne Arien in Hofkonzerten aufgeführt.

Das Fehlen von Werken Pietro Torris bereits im Katalog von 1806 spiegelt sich auch im Bestand der Bayerischen Staatsbibliothek wieder: Während zahlreiche der Opern und einige Gelegenheitswerke des über viele Jahre in München wirkenden Komponisten meist in autographen Partituren überliefert sind, ist hier keine einzige seiner Kirchenkompositionen mehr erhalten. ${ }^{17}$

Auch von den ursprünglich sehr zahlreich vorhandenen geistlichen Werken seines Nachfolgers Giovanni Porta, von dem 1806 noch 68 Stücke verzeichnet waren, ist heute nur noch wenig vorhanden: In dem 1860 an die Bibliothek abgegebenen Altbestand der Hofmusik-Intendanz befinden sich die Partituren zu zweien seiner Messen; ${ }^{18}$ zudem sind aus den Hofkapellbeständen aus dem Archiv der Theatinerkirche St. Kajetan kleinere liturgische Werke von ihm überliefert, nämlich acht Vertonungen des „Benedicat nos Deus“ (Mus.ms. Mk 717) und eine Gruppe von fünf Trauer-Antiphonen „ad tumulum“ (Mus.ms. Mk 716). Zu letzteren wurde noch um 1830 eine zusätzliche Cellostimme angefertigt, was belegt, dass diese Werke weiterhin zum Repertoire der Hofkapelle gehörten.

Die Kirchenmusik aus der Zeit der beiden letzten Kurfürsten aus der Linie der bayerischen Wittelsbacher, Karl Albrecht und Maximilian III. Joseph, ist insgesamt nur sehr lückenhaft überliefert. Neben dem Verlust zahlreicher Werke Torris und Portas fehlt auch die gesamte Kirchenmusik des Hofkapellmeisters Andrea Bernasconi; seine noch zu Robert Eitners Zeiten zahlreich vorhandenen Werke sind bei der Zerstörung der Allerheiligen-Hofkirche im Zweiten Weltkrieg verbrannt; sie konnten vermutlich nicht mehr rechtzeitig mit den übrigen Musikalien in die Theatinerkirche ausgelagert werden. Bernasconi, der in der Literatur bisher wegen seines konservativen Stils in seinen Opern überwiegend negativ beurteilt wurde ${ }^{19}$ scheint ein bedeutender Kir-

17 Im Werkverzeichnis bei Inga Mai Groote, Pietro Torri. Un Musicista veronese alla corte di Baviera, Verona 2003, S. 55-57, sind insgesamt 20 erhaltene geistliche Werke aufgeführt, die vornehmlich in Abschriften in Berlin, Brüssel und London überliefert sind, darunter als prominentestes Werk das Requiem für Kurfürst Maximilian II. Emanuel von 1726, B-Bc: 33889.

18 Missa Sta. Caeciliae C-Dur Mus.ms. 2474 (das Werk fehlt im Katalog von 1806, im alten Katalog findet sie sich als Nr. 14 von insgesamt 16 Messen) und Missa brevis B-Dur, Mus.ms. 2475.

19 Einige dieser abfälligen Bemerkungen zu Bernasconis Opernschaffen finden sich zusammengefasst in der Einleitung zu Daniela Sadgorski, Andrea Bernasconi und die Oper am Münchner Kurfürstenhof 1753-1772, München 2010, S. 1-8. 
chenkomponist gewesen zu sein, wie sein vor kurzem ediertes Miserere Nr. 7 in d-moll belegt. Der Mozart-Forscher John A. Rice rühmte das Werk in einem Vortrag als „masterpiece from Mozart's Munich “. ${ }^{20}$ Das Werk ist in Abschriften im Archiv des Doms von Passau sowie im Kloster St. Michael in Beromünster (Schweiz) erhalten. ${ }^{21}$ In der Passauer Quelle sind die Namen von zwei Münchener Sängern in die Stimmen eingetragen worden. ${ }^{22}$ Schriftvergleiche ergaben, dass es sich um Originalstimmen der Münchener Hofkapelle handelt, die vermutlich um 1780 vom Passauer Dom erworben wurden. ${ }^{23}$ Auf ähnlichem Wege gelangten wohl auch die Stimmen zu zwei Werken von Giovanni Porta nach Passau, einer Messe in C-Dur, die im Katalog der Kir-

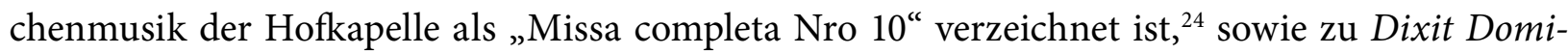
nus und Magnificat in C-Dur aus einem Vesperzyklus, verzeichnet im Katalog als „Dixit. Vesperae Nro 4 “ ${ }^{25}$ Erhalten sind dagegen sieben Kirchenmusikwerke von Joseph Willibald Michl (1745-1816), eines Schülers von Placidus Camerloher, der von 1770 bis zum Tode des Kurfürsten 1777 als „Cammercompositeur“ beim Hof angestellt war. ${ }^{26}$ Zudem hinterließen die meisten auswärtigen Komponisten, die für die jährlichen Karnevalsopern an den Münchener Hof engagiert wurden, eigene geistliche Werke für die Hofkapelle, die ebenfalls im Kirchenmusikkatalog von 1806 aufgeführt wurden. So schrieb Tommaso Traetta ein in München als autographe Partitur (Mus.ms. 2594) erhaltenes Stabat mater, während er anlässlich der Produktion seiner Oper Siroe 1767 in München weilte. Auch ein in München singulär überliefertes Laudate pueri von Antonio Sacchini (Mus.ms. 2516) dürfte im Zusammenhang mit einer der beiden Münchener Opern des Komponisten (L'Eroe cinese und Scipione in Cartagena) entstanden sein. Bekanntlich komponierte auch Wolfgang Amadeus Mozart ein geistliches Werk für die Hofkapelle, das Offertorium Misericordias Domini (KV 222), als er im Karneval 1775 seine Opera buffa La finta giardiniera in München aufführte.

Im Kontext des vorliegenden Bandes von Interesse sind schließlich noch einzelne Kirchenmusikwerke Dresdner Provenienz, die zum Bestand der Hofkapelle gehörten. Es handelt sich um ein heute in München nicht mehr erhaltenes Dixit Dominus in C-Dur von Joseph Schuster, ${ }^{27}$ sowie um die Abschriften des Requiems D-Dur für August den Starken von Jan Dismas Zelenka (ZWV 46) ${ }^{28}$ und der zum Namenstag von Maria Antonia Walpurgis 1760 von Johann Georg Schürer kompo-

20 „Andrea Bernasconi’s Miserere in D Minor: A Sacred Masterpiece from Mozart's Munich“, Referat von Christoph Riedo und John A. Rice, gehalten auf der Konferenz der Mozart Society of America, Medford/MA, 13. September 2015.

21 D-Po: Bernasconi 1, CH-BM: Mus.Ms.22.

22 Es handelt sich um den Tenor Johann Anton Berberich und um den Bassisten Giovanni Zonca.

23 Vergleiche hierzu das Vorwort zu der Edition des Werkes: Andrea Bernasconi, Miserere d-moll (Musik aus Schweizer Klöstern. 3), Adliswil/Zürich 2009, hrsg. von Christoph Riedo, S. III-IV.

24 D-Po: G. B. Porta 2. Vgl. Haberkamp und Münster, Die ehemaligen Musikhandschriftensammlungen (wie Anm. 6), S. 110.

25 D-Po: G. B. Porta 1. Vgl. ebd., S. 108.

26 Signaturen Mus.ms. 2357-2363. Die beiden Pange lingua in C und G sind neben den Stimmsätzen noch einmal separat als Autographe Mus.ms. 2718 und 2719 überliefert, beide datiert auf 1771, sie entstanden also zu Beginn von Michls Tätigkeit für den Münchener Hof.

27 Das Werk ist in Dresden unter der Signatur Mus.3549-E-544 überliefert. Vgl. das Incipit bei Haberkamp und Münster, Die ehemaligen Musikhandschriftensammlungen (wie Anm. 6), S. 116.

28 Mus.ms. 2706. 
nierten Missa solemnis in d-moll samt dem zugehörigen Offertorium Rogate quae ad pacem. ${ }^{29}$ Die Werke Zelenkas und Schürers wurden auf gleichem Papier und von dem gleicher Münchener Hofnotisten kopiert, dem vermutlich eine Dresdner Quelle als Vorlage diente. Schließlich gehörte auch ein Exemplar von Johann Gottlieb Naumanns Oratorium Isacco figura del redentore von 1772 zum Bestand der Hofkapelle, auch dieses Werk ist in einer Partiturabschrift erhalten (Mus.ms. 2372).

Die jüngeren in dem Kirchenmusikverzeichnis aufgeführten Werke von Ignaz Holzbauer, Paolo Grua, Joseph Georg Vogler, Peter von Winter und Franz Danzi, die der ,Mannheimer' Phase der Hofmusik unter Kurfürst Karl Theodor zuzurechnen sind, sind im Gegensatz zu den Kompositionen Bernasconis wieder sehr gut überliefert, das gleiche gilt für die Werke ihrer Nachfolger im 19. Jahrhundert. Diese Musikalien gelangten ebenfalls im Rahmen der Überführung der Noten aus der Hofmusik-Intendanz in den Besitz der Königlichen Bibliothek.

\section{Die Oper}

Unter der Regierung der Kurfürsten Ferdinand Maria und Maximilian II. Emanuel, vor des letzteren Abreise nach Brüssel im Jahr 1694, erlebte die Oper in München mit den Werken Giuseppe Antonio Bernabeis sowie des Hoforganisten und Kammermusikers Agostino Steffani eine außerordentliche Blüte. Allerdings ist diese glanzvolle Epoche der Münchener Operngeschichte nicht besonders gut in der Bayerischen Staatsbibliothek überliefert. Während die meisten der Libretti zu diesen prächtigen Hofopern in München erhalten sind, sind kaum noch Partituren aus dieser Zeit vorhanden. Interessanterweise finden sich aber Partituren von Münchener Opern und Serenaten von Steffani, Bernabei und dem jungen Pietro Torri heute in der Österreichischen Nationalbibliothek in Wien als Teil der Privatbibliothek („Bibliotheca cubicularis“) Kaiser Leopolds I. Sie könnten als Beutegut während der österreichischen Besatzung Münchens an den Wiener Hof gelangt sein, oder aus dem Erbe der in Wien verstorbenen Kurfürstin Maria Antonia (1669-1692) stammen, der ersten Gemahlin Maximilians II. Emanuel und Tochter Leopolds I.

Heute werden Partituren von Steffanis Münchener Opern Servio Tullio, Alarico il Baltha und Niobe, von Giuseppe Antonio Bernabeis L'Ascanio und Il Segreto d'amore in petto del Savio, sowie von zwei frühen Serenaten Pietro Torris, Fetonte und I pregi della primavera in der Bayerischen Staatsbibliothek verwahrt, sie gehören zu der als Depositum in München aufbewahrten Sammlung der Grafen Toerring-Jettenbach. Da sie aus dem Privatbesitz des Kölner Erzbischofs Joseph Clemens (1671-1723) stammen, des jüngeren Bruders von Kurfürst Max Emanuel, für den Torri später auch zahlreiche Werke komponieren sollte, sind es Handschriften, die dem engsten familiären Umfeld des Münchener Hofes zuzurechnen sind. ${ }^{30}$

Der Vizekapellmeister Pietro Torri, der später Bernabei als erster Kapellmeiter nachfolgte, gehörte zum Hofstaat des Kurfürsten auf dessen erstem Brüsseler Aufenthalt als Statthalter der

29 Mus.ms. 2537 (Missa) / 2538 (Offertorium).

30 Gertraut Haberkamp und Barbara Zuber: Die Musikhandschriften Herzog Wilhelms in Bayern, der Grafen zu Toerring-Jettenbach und der Fürsten Fugger von Babenhausen. Thematischer Katalog [mit einem Vorwort zur Geschichte und Inhalt der Sammlung von Robert Münster] (Kataloge Bayerischer Musiksammlungen. 13), München 1988, S. XXIII. 
Spanischen Niederlande im Jahr 1694. Auch während der Exiljahre Max Emanuels in Brüssel und in Nordfrankreich zwischen 1704 und 1715 diente Torri als Kapellmeister vor Ort, während Bernabei auch während der österreichischen Besatzung in München blieb, wo er weiterhin für die Musikpflege an der Hofkirche zuständig war. An Torri-Autographen besitzt die Bibliothek einen reichen Bestand aus 16 Opern, elf Serenaten und Turnier-Musiken („tornei“) und zehn Oratorien. Die Opern entstanden mit vier Ausnahmen alle erst nach der Rückkehr Maximilian Emanuels aus dem Exil 1715, einige auch erst nach dem Tod dieses Kurfürsten und dem Regierungsantritt seines Sohnes Karl Albrecht. Drei der erhaltenen Bühnenwerke schrieb Torri für den ebenfalls vor den Österreichern geflohenen Joseph Clemens, der einen Exilhof im nordfranzösischen Valenciennes unterhielt. Es handelt sich um die Pasticcio-Oper Le Peripezie della Fortuna (Mus.ms. 209), die neben Musik Torris auch einige Arien aus Opern Agostino Steffanis enthält, eine einaktige komische Oper in französischer Sprache, Le Réciproque (Mus.ms. 243), und die sechsaktige Festoper L'innocenza difesa da' numi (Mus.ms. 212). Das vierte Werk ist die für Hannover entstandene Oper Briseide von 1696, die Torri vermutlich auf Vermittlung seines Lehrers Agostino Steffani komponierte.

Geistliche Gegenstücke zu den Opern sind die zehn in München überlieferten Oratorien Torris. Darunter befinden sich wieder einige Werke, die für Joseph Clemens in Valenciennes komponiert wurden. ${ }^{31}$ Dass sich unter den Münchener Torri-Autographen so zahlreiche Werke befinden, die nicht in München uraufgeführt wurden, lässt vermuten, dass diese Partituren aus dem Nachlass des 1737 verstorbenen Komponisten stammen könnten.

Opern anderer Komponisten, die während der Regierungszeit Karl Albrechts aufgeführt wurden, fehlen hingegen zum größten Teil in der Münchener Sammlung, nur noch Abschriften der Clemenza di Tito von Francesco Peli von 1736 (Mus.ms. 161) und von Giovanni Portas Ifigenia in Aulide von 1739 (Mus.ms. 20887, Partitur aus der Sammlung der Kurfürstin Maria Anna) sind in München überliefert, beide Opern sind bezeichnenderweise auch in der Sammlung Maria Antonias in Dresden erhalten.

Auch die nach dem Regierungsantritt Maximilian III. Josephs komponierten Opern des Kammerkomponisten Giovanni Ferrandini sind mit Ausnahme seiner Vertonung von Maria Antonias Talestri-Libretto von 1760 (Mus.ms. 199) nicht mehr in der Münchener Sammlung erhalten. Umso wertvoller ist die relativ gute Überlieferung seiner Werke in der Sammlung Maria Antonias, darunter die Vertonung von Metastasios Catone in Utica, das Werk, mit dem das von Cuvilliés erbaute Opernhaus in der Residenz 1753 eröffnet wurde. ${ }^{32}$ Die Opern von Portas Nachfolger Andrea Bernasconi sind dagegen mit Ausnahme des frühen Agelmondo vollständig überliefert. Es ist nicht mehr ganz eindeutig zu ermitteln, aus welchem Bestand die einzelnen prachtvoll eingebundenen Partituren stammen, zumal in einigen Fällen auch Dubletten vorliegen. Einige daraus gehören mit großer Sicherheit zu der weiter unten beschriebenen Privat-Musikaliensammlung der Kurfürstin Maria Anna.

31 Zur korrekten Datierung und Zuschreibung der Werke siehe Barbara Zuber, „Pietro Torri und das Wittelsbacher Musiktheater im Exil“, in: Das Musikleben am Hof von Kurfürst Max Emanuel, hrsg. von Stephan Hörner und Sebastian Werr, Tutzing 2012, S. 127-169; vgl. vor allem die Übersicht der erhaltenen Libretti in Anhang 1, S. 164-166. 
Seit dem späten 18. Jahrhundert, also den letzten Regierungsjahren Carl Theodors, sind zahlreiche Aufführungsmaterialien der Bayerischen Staatstheater erhalten, die sich vor ihrer Eingliederung in die Musikabteilung der Bayerischen Staatsbibliothek im Archiv der Staatsoper befanden. Diese häufig sehr heterogen aus Drucken (Klavierauszüge und Partituren) und Handschriften (Stimmen und Partituren, Einlagearien, Souffleurbücher) zusammengesetzten Materiale sind nicht in die Aufstellung der Musikhandschriften (Mus.ms.) aufgenommen worden, sondern bilden eine eigene Signaturengruppe St.th. Die Bestände sind im Opac der Bayerischen Staatsbibliothek nachgewiesen und können über eine eigene Suchmaske als „Historisches Aufführungsmaterial der Bayerischen Staatsoper" recherchiert werden. ${ }^{33}$ Eine Übernahme der Dateien in den RISM-Katalog wurde bisher nicht vorgenommen, anders als im Falle der vergleichbaren Bestände der Sächsischen Hofoper in Dresden, deren hervorragende, im Rahmen von DFG-Projekten angefertigte Katalogisate eine wertvolle Bereicherung der RISM-Datenbank darstellen.

\section{Privatsammlungen}

Anders als bei den Wettinern in Dresden, sind bei den bayerischen Wittelsbachern nur wenige Quellen dem Privatbesitz eines bestimmten Fürsten oder einer Fürstin des Herrscherhauses zuzuweisen, da entsprechende Kataloge mit einer Ausnahme fehlen. Geschlossene größere PrivatMusikaliensammlungen, die in den Bestand der Bibliothek übernommen wurden, sind erst für das 19. Jahrhundert überliefert, etwa die Sammlungen der Könige Maximilian II. (die berühmte Collectio musicalis Maximilianea), Ludwig II. oder des ersten Königs von Griechenland Otto I.

Trotzdem können in Einzelfällen Musikalien früheren Besitzern aus der Familie des Herrscherhauses zugewiesen werden. Ein Beispiel aus dem 17. Jahrhundert stellt die Gitarrentabulatur Mus.ms. 1522 dar, die Adelaide von Savoyen, der Gattin des Kurfürsten Ferdinand Maria, gehörte. ${ }^{34}$ Bedeutender sind die Reste der Notensammlung der ersten Gemahlin von Maximilian II. Emanuel, der früh verstorbenen Erzherzogin Maria Antonia, der Tochter seines späteren Kontrahenten Leopolds I. Schon Maier wies in seinem gedruckten Katalog darauf hin, dass einige der Handschriften des späten 17. Jahrhunderts aus ihrem Besitz stammen müssten. ${ }^{35}$ Es handelt sich ausschließlich um Sammlungen von Werken für Singstimmen und Basso continuo, neben deutschen Liedern vor allem Kollektionen mit italienischen Kantaten ${ }^{36}$ und Opernarien. Eine Sensation stellt vor allem eine zweibändige anonyme Ariensammlung dar, bei der es sich, wie sich im Rahmen der Erschließung für RISM herausstellte, um eine vollständige Übertragung der Arien des ersten und dritten Aktes der Oper Alarico il Baltha von Agostino Steffani handelt. ${ }^{37}$ Die Oper war 1687 zum Geburtstag der jungen Kurfürstin aufgeführt worden, so dass die Zuordnung der beiden Bände zu ihrer Privatsammlung plausibel erscheint. Aus Maria Antonias Heimatstadt Wien stammen drei Bände mit Ausschnitten aus Opern Antonio Draghis, des Kapellmeisters Leopolds I. Der

33 https://hadbso.bsb-muenchen.de (25.10.2016).

34 Hierzu Maier, Die Musikalischen Handschriften (wie Anm. 7), S. 148.

35 Ebd., S. 140-141.

36 Mus.ms. 1510 und Mus.ms. 1527.

37 Mus.ms. 1517 (Akt 1) und Mus.ms. 1518 (Akt 2). 
erste Band (Mus.ms. 1519) enthält Stücke aus der Oper Il Silentio di Arpocrate von 1677, die noch einmal 1688 zum Namenstag des Kaisers von Damen und Kavalieren des Wiener Hofes aufgeführt wurde; die Abschrift der Sammlung entstand vermutlich anlässlich dieser Wiederaufführung. Im zweiten Band (Mus.ms. 1523) befinden sich Arien aus den in Augsburg anlässlich der Königswahl Josephs I. aufgeführten Festopern La Regina de Volsci und Telemaco, wie im Titel mitgeteilt wird: „Ariette delle due Opere cantate in Augusta nel tempo dell'elezione di Giuseppe I in Rè de Romani composte dal S|e Antonio Draghi, Maestro di Capella di S. M. ${ }^{\text {tà }}$ Cesarea, negl'anni 1689 e 1690 “. Der dritte Band (Mus.ms. 1520) vereint Arien aus den Opern Gli Essercitii poetici und La Vittoria della fortezza. Alle Bände enthalten auch Einlagearien von Leopold I., die dieser zu den Partituren seines Hofkapellmeisters beisteuerte; sie sind in den Bänden mit der Abkürzung „S. M. C.“ (Sua Maestà Cesarea) gekennzeichnet. Wiener Provenienz ist auch der autographe Sammelband mit deutschen Generalbassliedern von Johann Jacob Prinner, der eine Widmungsvorrede an die Kurfürstin enthält (Mus.ms. 1577). Ein Bleistiftvermerk in dem Band verrät, dass die Noten aus dem Besitz von Graf Josef Ferdinand Maria von Salern (1718-1805) stammen, dem Generalintendanten der Hofmusik unter Maximilian III. Joseph. Sie gelangten erst im späteren 19. Jahrhundert als Geschenk des berühmten Grafen Franz von Pocci in den Besitz der Königlichen Bibliothek.

Aus dem Besitz der zweiten Gattin Max Emanuels, der polnischen Prinzessin Therese Kunigunde, die während der österreichischen Besatzung Münchens im Exil in Venedig lebte, ist nur eine Handschrift erhalten. Es handelt sich um eine Sammelhandschrift mit zwölf Kantaten (Signatur Mus.ms. 3189). Neben anonymen Werken enthält der von dem Kopisten Antonio Passarini geschriebene Band Stücke von Alessandro Scarlatti, Francesco Mancini, Pietro Bencini und Emanuele d'Astorga. Sie konnte von Berthold Over eindeutig dem Besitz der Kurfürstin zugewiesen werden und muss während ihres Venedig-Aufenthaltes entstanden sein. ${ }^{38}$ Leider ist von der durch Over mittels Archivstudien rekonstruierten Musikaliensammlung Therese Kunigundes, die vor allem Abschriften von Opern umfasste, sonst keine einzige Handschrift mehr in München nachweisbar.

Auch die vierte, hier als letztes zu betrachtende Teilsammlung gehörte einer Kurfürstin. Hier schließt sich wieder der Kreis nach Dresden, denn es handelt sich um Max Josephs sächsische Gattin Maria Anna, die Schwester Friedrich Christians. Ihre bereits erwähnte Sammlung ist als einzige der älteren Privatsammlungen durch einen erhaltenen thematischen Katalog erschlossen und kann deshalb in Grundzügen rekonstruiert werden. Dieser Catalogo de Libri di Musica di S. A.S. E. Maria Anna Elettrice befand sich im Besitz des Musikgelehrten Karl Emil von Schafhäutl (1803-1890), der seine wertvolle Musikaliensammlung 1864 der Königlichen Bibliothek schenkte. Der Katalog dieser Sammlung wurde zusammen mit dem handschriftlichen Katalog der Kirchenmusik der Hofkapelle von Gertraud Haberkamp und Robert Münster ediert. ${ }^{39}$ Er enthält fast ausschließlich Vokalmusik, darunter Ariensammlungen sowie vollständige Partituren von Opern, Oratorien, Kantaten und Serenaten. In dem Katalog sind auch zahlreiche Werke Dresdner Provenienz aufgeführt, an erster Stelle stehen dabei Abschriften von Opern Johann Adolf Hasses.

38 Siehe hierzu Berthold Over, „Kurfürstin Therese Kunigunde von Bayern in Venedig (1705-1715)“, in: Das Musikleben am Hof von Kurfürst Max Emanuel, hrsg. von Stephan Hörner und Sebastian Werr, Tutzing 2012, S. 85-117, hier S. 93-96.

39 Siehe Anm. 4. Die Signatur des Katalogs: Mus.ms. 1648. 
Daneben sind auch Werke im Katalog enthalten, die während der Doppelhochzeit von 1747 in Pillnitz und Dresden erklangen, die Serenaten Le Nozze di Ercole ed Ebe von Gluck ${ }^{40}$ und Galatea von Schürer sowie dessen Oper Ercole sul Termodonte. Von jüngeren Dresdner Musikern sind italienische Duette von Naumann sowie das Oratorium La Passione von Joseph Schuster im Katalog nachgewiesen.

Von den in München aufgeführten Opern wurden neben dem Exemplar für die HofmusikIntendanz weitere Kopien für Angehörige des Herrscherhauses angefertigt. Robert Münster konnte dies etwa an Antonio Tozzis Orfeo belegen, der auch im Katalog der Sammlung Maria Annas nachgewiesen ist. ${ }^{41}$ Aufgrund dieser Gepflogenheit sind die meisten in München zu Lebzeiten ihres Gatten aufgeführten Karnevalsopern auch in der Kollektion der Fürstin vertreten. Dagegen sind nur noch zwei der in der folgenden Regierungszeit Carl Theodors gespielten Bühnenwerke im Inventar verzeichnet: Franz Paul Gruas Telemaco (1780) und Alessio Pratis Armida (1785); eine Abschrift der berühmtesten Münchener Karnevalsoper, Mozarts Idomeneo, fehlte in ihrer Sammlung.

Nur ein Teil der Partituren und Sammelhandschriften aus dem Besitz der Kurfürstin ist erhalten. Einige der Bände befanden sich vormals im Besitz von Johann Michael Hauber und von Caspar Aiblinger, dem Münchener Kapellmeister, und gelangten also erst im Verlauf des 19. Jahrhunderts in den Besitz der Königlichen Bibliothek. Weitere Bände befanden sich bis vor kurzem in der Bibliothek der Münchener Musikhochschule, die als Nachfolgeinstitution des „Königlichbayerischen Conservatoriums für Musik“ diese Quellen verwahrte; sie wurden inzwischen an die Musikabteilung der Bayerischen Staatsbibliothek abgegeben. Von besonderem Wert ist dabei ein Band mit 32 Einzelwerken von Giovanni Ferrandini, dessen Werke ansonsten in München nur sehr lückenhaft überliefert sind. ${ }^{42}$ Sechs Handschriften aus dem Besitz der Kurfürstin - darunter Bernasconis Oratorium Betulia liberata - konnte Gertraud Haberkamp in der Österreichischen Nationalbibliothek nachweisen, ein Sammelband mit Arien aus der römischen Oper Farnace von Davide Perez gelangte dagegen in die Hamburger Staatsbibliothek. ${ }^{43}$ Dies zeigt, dass ihre Sammlung wohl nach ihrem Tod zunächst verstreut wurde.

Die Ähnlichkeit in Aufbau und Zusammensetzung der Sammlung Maria Annas mit derjenigen ihrer Schwägerin Maria Antonia Walpurgis lässt vermuten, dass sie eine ähnliche Funktion erfüllte. Die in ihr enthaltenen Werke erklangen wohl vorwiegend den höfischen Kammerkonzerten, in denen sich die Angehörigen der kurfürstlichen Familie gemeinsam mit Mitgliedern der Hofkapelle sowie mit gastierenden Musikern hören ließen, so wie es Charles Burney während seines zu Beginn erwähnten Besuches 1772 in der bayerischen Residenzstadt erleben konnte.

40 Erhalten in München, Signatur Mus.ms. 530.

41 Haberkamp und Münster, Die ehemaligen Musikhandschriftensammlungen (wie Anm. 6), S. XXII. Das Exemplar für Maria Antonia Walpurgis ist in Dresden erhalten: D-Dl: Mus.3398-F-1, das Münchner Exemplar Mus. ms. 2592 stammt aus den Beständen der Hofmusik-Intendanz.

43 Haberkamp und Münster, Die ehemaligen Musikhandschriftensammlungen (wie Anm. 6), S. XXII. 